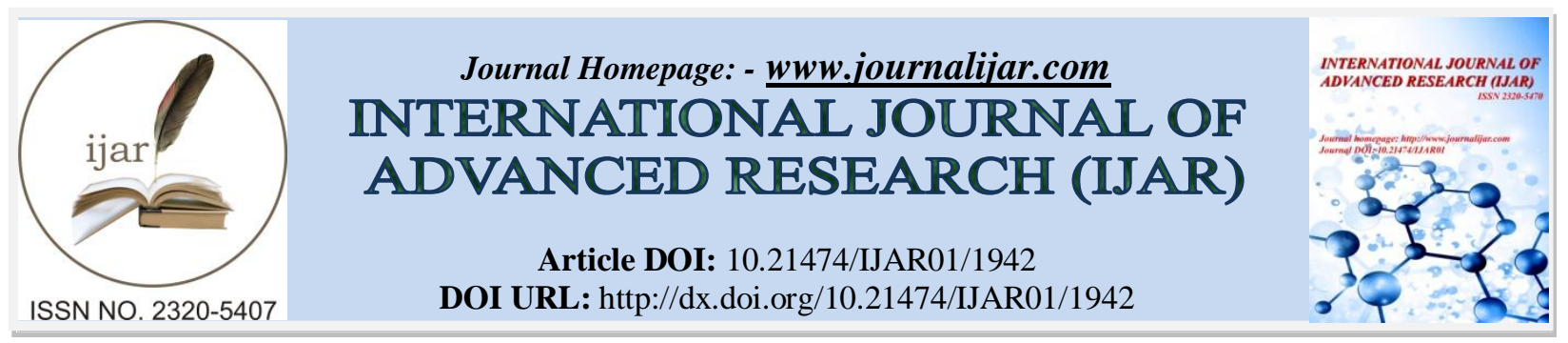

RESEARCH ARTICLE

\title{
ANTI-BACTERIAL AND ANTI-TUBERCULAR STUDIES OF SOME NOVEL TRIAZINONE DERIVATIVES.
}

Chandraprabha. V. J*1, Jagadeesh Prasad $D^{1}$ and Prashantha Nayak ${ }^{3}$.

1. Department of Chemistry, Mangalore University, Mangalagangotri, Karnataka-574199.

2. Department of Bioscience, Mangalore University, Mangalagangotri, Karnataka-574199.

\section{Manuscript Info}

Manuscript History

Received: 11 August 2016

Final Accepted: 22 September 2016

Published: October 2016

Key words:-

Triazinones, anti-TB, anti-inflammatory, anti-bacterial activities, spectroscopic data.

\section{Abstract}

Triazinones and its derivatives are one of the versatile compounds which show significant importance because of its enormous biological activities. The present study deals with the preparation of series of novel [3-tert-butyl-7-(aryl)-4H-[1,3,4]thiadiazolo[2,3-c][1,2,4]triazin4-one] (5a-k) (Scheme -2.1) from condensation of [4-amino-6-tertbutyl-3-sulfanyl-1,2,4-triazin-5(4H)-one] (3) and different substituted aryloxy acetic acids (4). The structures of these novel compounds were confirmed and characterized by elemental analysis, FT-IR, ${ }^{1} \mathrm{H}-$ NMR, ${ }^{13 C}$-NMR, LC-Mass. They were also screened in vitro for their anti-TB, anti-inflammatory and anti-bacterial activities.

Copy Right, IJAR, 2016,. All rights reserved.

\section{Introduction:-}

Triazinones scaffolds are important class of heterocyclic chemistry due to their unique biological properties. The advancement in the current research and output of literature studies reveals that triazinone and its derivatives show attractive biological activities such as, anti-microbial ${ }^{1}$, anti- tumour $^{2}$, anti-tubercular ${ }^{3}$, anti-bacterial ${ }^{4}$, anti-convulsant agents $^{5}$, anti-cancer ${ }^{6}$, anti-proliferative ${ }^{7}$, anti-metastatic ${ }^{8}$, anti- $\mathrm{HIV}^{9}$, anti-depressant ${ }^{10}$, anti-inflammatory ${ }^{11}$, analgesic $^{11}$, ulcerogenic ${ }^{11}$.In addition to these activities triazinones are also used as, fungicidal ${ }^{12}$, Pesticidal ${ }^{13}$, mosquito-larvicidal and insecticidal agents ${ }^{14-17}$. Marching in the same direction it was observed that Triazinone derivatives drew considerable interest.

Therefore, the literature review of triazinones lead to the idea of prominent reactions schemes and products that reflects the importance of the triazinone derivatives, hence we synthesized the novel [3-tert-butyl-7-(aryl)- $4 \mathrm{H}$ $[1,3,4]$ thiadiazolo[2,3-c] $[1,2,4]$ triazin-4-one $](5 \mathrm{a}-\mathrm{k})$ and screened for their anti-TB, anti-inflammatory and antibacterial activities.

\section{Materials and Methods:-}

4-amino-6-tert-butyl-3-sulfanyl-1, 2, 4-triazin-5(4H)-one] (3) was synthesized according to Dornow and co-workers procedure ${ }^{18-21}$. The equimolar mixture of 3,3-dimethyl-2-oxobutanoic acid (trimethyl pyruvic acid) and thiocarbohydrazide (2) were refluxed in ethanol for 12 hours, to yield [4-amino-6-tert-butyl-3-sulfanyl-1,2,4triazin-5(4H)-one] (3) which was later condensed with different substituted aryloxy acetic acids (4) in $\mathrm{POCl}_{3}$ at $90^{\circ} \mathrm{C}$ for 8 hours to yield series of novel [3-tert-butyl-7-(aryl)-4H-[1,3,4]thiadiazolo[2,3-c][1,2,4]triazin-4-one] (5ak) (Scheme -1). The structures of these novel compounds (5a-k) were confirmed through spectral analysis and the data are incorporated in the experimental section.

Corresponding Author:- Chandraprabha. V. J.

Address:- Department of Chemistry, Mangalore University, Mangalagangotri, Karnataka-574199. 
Melting points of the compounds (5a-k) were determined in open capillary tubes and are uncorrected. The purity of synthesized compounds was checked by TLC observing single spot on Merck silica gel $60 \mathrm{~F}_{254}$ coated alumina plates. The structures of these novel compounds $(5 \mathrm{a}-\mathrm{k})$ were confirmed through spectral studies. The IR spectra $\left(\mathrm{cm}^{-}\right.$ ${ }^{1}$ ) were recorded on a Shimadzu-FTIR 577 infrared spectrometer in $\mathrm{KBr}$ pellets. The ${ }^{1} \mathrm{H}-\mathrm{NMRand}{ }^{13} \mathrm{C}-\mathrm{NMR}$ spectra was recorded on Brucker AMX-400(400MHz) spectrometer using $\mathrm{CDCl}_{3}-\mathrm{d}$ as solvent and TMS as the internal standard. The mass spectra were recorded on Perkin -Elmer 018444Y, triple quadrapole LC/MS spectrometer. The synthesized novel compounds showed the molecular ion peak $(\mathrm{m} / \mathrm{z})$ equivalent to their molecular weight. The synthetic pathways of the studies are indicated in Scheme 1 in spectroscopic data. The characterization data and biological activity data are tabulated in Table1, 2 and 3 respectively.

Reaction scheme:-<smiles>CC(C)(C)C(=O)C(=O)O</smiles>

[1]<smiles>CCOC(C)CC(C)CC</smiles>

[2]<smiles>[R]c1ccccc1C(=O)O</smiles>

[3]

[4]<smiles>[R]c1cccc(-c2nn3c(=O)c(C(C)(C)C)nnc3s2)c1</smiles>

$$
\begin{gathered}
\mathrm{R}=2,3,4-(\mathrm{OMe})_{3}, 2-\mathrm{Me}, 3-\mathrm{NO}_{2}, 3-\mathrm{Ph} .4-\mathrm{N} ; 2-\mathrm{COC}_{6} \mathrm{H}_{5} \mathrm{Cl}, 2-\mathrm{Cl}, 5-\mathrm{Br}, \\
2-\mathrm{Br}, 5-\mathrm{Cl}, 3,5-\mathrm{Br}_{2}, 4-\mathrm{OCH}\left(\mathrm{CH}_{3}\right)_{2}, 4-\mathrm{CF}_{3}, 2-\mathrm{F}, 5-\mathrm{NO}_{2} ; 4-\mathrm{CH}\left(\mathrm{CH}_{3}\right)_{2}
\end{gathered}
$$

(Scheme-2.1)

\section{General procedure for the synthesis of 4-amino-6-tert-butyl-3-sulfanyl-1, 2, 4-triazin-5(4H)-one (3):-}

According to well described procedure in literate the synthesis of 4-amino-6-tert-butyl-3-sulfanyl-1, 2, 4-triazin$5(4 \mathrm{H})$-one (3) was carried out. The equimolar mixture of 3, 3-dimethyl-2-oxobutanoic acid (trimethyl pyruvic acid) [CAS no: 815-17-8] (1) and thiocarbohydrazide (2) were refluxed in ethanol solvent for 12 hours. The purity and completeness of the reaction was monitored through TLC, the compound (3) was precipitated by adding crushed ice into the reaction mixture which is later dried and recrystallized from ethanol. Yield and melting points were noted. 
General procedure for the synthesis of 3-tert-butyl-7-(aryl)-4H [1,3,4]thiadiazolo[2,3-c][1,2,4]triazin-4-one (5a-k).

The equimolar mixture of [4-amino-6-tert-butyl-3-sulfanyl-1,2,4-triazin-5(4H)-one] (0.01 mol) (3) and different substituted aryloxy acetic acids $(0.01 \mathrm{~mol})$ (4) were condensed in the presence of $\mathrm{POCl}_{3}$ at $90^{\circ} \mathrm{C}$ for 8 hours under dry condition. The reaction mixtures were cooled and poured into crushed ice drop wise with vigorous shaking, the solid product separated which was later filtered and recrystallized from ethanol to get series of novel target compounds (5a-k) (Scheme-2.1).

\section{Results and Discussions:-}

The novel 3-tert-butyl-7-(aryl)-4H [1,3,4]thiadiazolo[2,3-c][1,2,4]triazin-4-one (5a-k).

Were synthesized by dehydration and cyclization of [4-amino-6-tert-butyl-3-sulfanyl-1, 2, 4-triazin-5(4H)-one] and substituted aryloxy acetic acids In the presence of $\mathrm{POCl}_{3}$. The presence of carbonyl stretch of triazinone ring and the absence of absorption band due to $-\mathrm{NH}_{2}$ group in the product, confirms the involvement of $\mathrm{N}-\mathrm{NH}_{2}$ of the triazinone ring in the formation of thiadiazolotriazinone. The formation of the novel product was confirmed by the FT-IR, ${ }^{1} \mathrm{H}-$ NMR and ${ }^{13} \mathrm{C}$-NMR. The LC-Mass signals for molecular ion were consistent with its molecular formula.

\section{Spectroscopic data:-}

(5a): IR $\left(\mathrm{KBr}, \mathrm{Cm}^{-1}\right)$ : 2955.51 ( $>\mathrm{C}-\mathrm{H}$ stretch, $-\mathrm{CH}_{3}$ groups of tert butyl moiety and $-\mathrm{CH}_{3}$ groups attached to1,2,4,triazin-4-one),1591.95 ( $>\mathrm{C}=0$, , 2,4,-triazin-4-one), 1591.95, 1514.59, 1413.26, $1361.33(>\mathrm{C}=\mathrm{N},>\mathrm{C}=\mathrm{C}<$ stretch), $1491\left(>\mathrm{N}-\mathrm{N}<\right.$ stretch) and 1331.59 (>C-S- stretch). ${ }^{1} \mathrm{H}-\mathrm{NMR}:\left(\mathrm{CDCl}_{3} \delta \mathrm{ppm}\right): 1.195\left(9 \mathrm{H}, \mathrm{s}, 3 \mathrm{CH}_{3}\right.$ groups ,tert butyl moiety), 3.914, 3.974, 4.118 (9H, S, 3OCH 3 groups), 6.849 ( $1 \mathrm{H}, \mathrm{d}, \mathrm{J}=8.8$ 2,3,4- methoxy phenoxy moiety), 8.166 $\left(1 \mathrm{H}, \mathrm{d}, \mathrm{J}=9.2\right.$ 2,3,4- methoxy phenoxy moiety). ${ }^{13} \mathrm{C}-\mathrm{NMR}: 27.736\left(3 \mathrm{CH}_{3}\right.$ groups,ter butyl moiety), 38.018 (quaternary $\mathrm{C}$ atom, tert butyl moiety),56.322, 60.995, 61.287(3C atoms of 3- methoxy groups), 108.220, 114.018, $123.722,141.534,147.093,152.659$ (6C atoms of 2,3,4-methoxy phenoxy group), 156.006, 158.083, 159.910 (3C atoms thiadiazolotriazin-4-one moiety),160.924(>C=0 thiadiazolotriazin-4-one).LC-Mass: [ $\left.\mathrm{M}^{+}+1\right],(\mathrm{m} / \mathrm{z}): 377.01$.

(5b):IR ( $\left.\mathrm{KBr}, \mathrm{Cm}^{-1}\right)$ : 3083.67(>C-H stretch, 2-methyl-3-nitro phenoxy), 2980.57, 2934.02 (>C-H stretch, $-\mathrm{CH}_{3}$ groups of tert butyl moiety and $-\mathrm{CH}_{3}$ groups attached to1,2,4,-triazin-4-one), 1710.43 ( $>\mathrm{C}=0,2,4$,-triazin-4-one ),1540.99, ( $>\mathrm{C}=\mathrm{N}), 1513.92,1320.50$ ( $>\mathrm{C}=\mathrm{C}<$ stretch), 1456.76(>N-N $<$ stretch), 1320.50 (>C-S- stretch), 783.92 (-C-Br stretch) and 1540.97, 1513.92 (symmetric and asymmetric stretch of $-\mathrm{NO}_{2}$ group). ${ }^{1} \mathrm{H}-\mathrm{NMR}:\left(\mathrm{CDCl}_{3} \delta\right.$ ppm):1.915(9H, s, $3 \mathrm{CH}_{3}$ groups, tert butyl moiety), $7.243(1 \mathrm{H}, \mathrm{d}, \mathrm{J}=8.2$, 2-methyl-3-nitrophenyl), $8.176(1 \mathrm{H}, \mathrm{d}, \mathrm{J}=8$ 2-methyl-3-nitrophenyl), 7.989(1H, t, 2-methyl-3-nitrophenyl). ${ }^{13} \mathrm{C}-\mathrm{NMR}: 27.925\left(3 \mathrm{CH}_{3}\right.$ groups,ter butyl moiety), 38.779 (quaternary $\mathrm{C}$ atom, tert butyl moiety),108.220, 114.018, 123.722, 141.534, 147.093, 152.659 (6C atoms of 2-methyl-3-nitrophenoxy group),156.006, 158.083, $159.910 \quad$ (3C atoms thiadiazolotriazin-4-one moiety),160.992(>C=0 thiadiazolotriazin-4-one).LC-Mass: [ $\left.\mathrm{M}^{+}+1\right],(\mathrm{m} / \mathrm{z}): 345.37$.

5c:IR $\left(\mathrm{KBr}, \mathrm{Cm}^{-1}\right): 3115.43,3078.27(>\mathrm{C}-\mathrm{H}$ stretch, quinoline and phenyl group) ,2760.72, 2925.92 (>C-H stretch, $\mathrm{CH}_{3}$ groups of tert butyl moiety and $-\mathrm{CH}_{3}$ groups attached to1,2,4,-triazin-4-one), 1693.17 (>C=0, 2,4,-triazin-4-one ), $1510.92(>\mathrm{C}=\mathrm{N}$ stretch), 1543.20,1383.33,1360.73( $>\mathrm{C}=\mathrm{C}<$ stretch), 1449.38( $>\mathrm{N}-\mathrm{N}<$ stretch) and $1287.56(>\mathrm{C}-\mathrm{S}-$ stretch). ${ }^{1} \mathrm{H}-\mathrm{NMR}:\left(\mathrm{CDCl}_{3} \delta \mathrm{ppm}\right) 1.185\left(9 \mathrm{H}, \mathrm{s}, 3 \mathrm{CH}_{3}\right.$ groups, tert butyl moiety), $8.176(1 \mathrm{H}, \mathrm{d}, \mathrm{J}=8$, quinoline moiety), $8.234(1 \mathrm{H}, \mathrm{d}, \mathrm{J}=8$, quinoline moiety), $8.567(2 \mathrm{H}, \mathrm{m}$ quinoline moiety).7.945(1H,s, quinoline moiety), 7.956 $(2 \mathrm{H}, \mathrm{d}, \mathrm{J}=8.1$, phenyl ring attached to quinoline moiety), $8.112(2 \mathrm{H}, \mathrm{d}, \mathrm{J}=8$, phenyl ring attached to quinoline moiety), 8.346 $\left(1 \mathrm{H}, \mathrm{s}\right.$, phenyl ring attached to quinoline moiety) ${ }^{13} \mathrm{C}-\mathrm{NMR}: 27.996\left(3 \mathrm{CH}_{3}\right.$ groups, ter butyl moiety), 38.787 (quaternary $\mathrm{C}$ atom, tert butyl moiety), 109.210, 109.154,110.254, 115.918, 123.975, 130.763, 131.845, $134.145,135.023,136.789,140.879,141.556,141.654,148.235,153.679$ (15C atoms of quinoline and phenoxy group), 157.134, 158.188, 159.092 (3C atoms thiadiazolotriazin-4-one moiety) $161.196(>\mathrm{C}=0$ thiadiazolotriazin-4one).LC-Mass: $\left[\mathrm{M}^{+}+1\right],(\mathrm{m} / \mathrm{z}): 413.537$.

(5d):IR (KBr, $\left.\mathrm{Cm}^{-1}\right): 3115.43,3078.27(>\mathrm{C}-\mathrm{H}$ stretch, [(4chlorophenyl)carbonyl] phenoxy) ,2754.72, $2946.52(>\mathrm{C}-\mathrm{H}$ stretch, $-\mathrm{CH}_{3}$ groups of tert butyl moiety and $-\mathrm{CH}_{3}$ groups attached to1,2,4,-triazin-4-one), 1723.17,1556.09 ( $>\mathrm{C}=0$, 2,4,-triazin-4-one and 4-chlorophenyl ),1520.52 ( $>\mathrm{C}=\mathrm{N}$ stretch),1533.20,1363.33,1380.73( $>\mathrm{C}=\mathrm{C}<$ stretch), 1449.38(>N-N< stretch), 1299.56 (>C-S- stretch) and 832.40(C-Cl stretch). ${ }^{1} \mathrm{H}-\mathrm{NMR}:\left(\mathrm{CDCl}_{3} \delta \mathrm{ppm}\right): 1.9175(9 \mathrm{H}, \mathrm{s}$, $3 \mathrm{CH}_{3}$ groups, tert butyl moiety), 7.214 $(2 \mathrm{H}, \mathrm{d}, \mathrm{J}=8$, 4-chlorophenoxy carbonyl), $8.312(2 \mathrm{H}, \mathrm{d}, \mathrm{J}=8.2$, phenyl ring adjacent to thiadiazolotriazin-4-one ), $8.372(2 \mathrm{H}, \mathrm{d}, \mathrm{J}=8$, phenyl ring adjacent to thiadiazolotriazin-4-one) , $8.324(2 \mathrm{H}, \mathrm{m}$, phenyl ring adjacent to thiadiazolotriazin-4-one $)$.

${ }^{13} \mathrm{C}-\mathrm{NMR}: 27.894\left(3 \mathrm{CH}_{3}\right.$ groups,ter butyl 
moiety), 38.737 (quaternary $\mathrm{C}$ atom, tert butyl moiety), 109.210, 110.365, 115.918,116.762, $123.975,126.276,130.156,132.076, \quad 138.564, \quad 141.654, \quad 148.235, \quad 153.679 \quad(12 \mathrm{C}$ atoms of 2-[(4chlorophenyl)carbonyl]phenoxy group),157.134, 158.188, 159.092 (3Catoms thiadiazolotriazin-4-one moiety),161.196, 160.453 (>C=0 thiadiazolotriazin-4-one and 4-chlorophenoxy carbonyl ).LC-Mass: $\left[\mathrm{M}^{+}+1\right],(\mathrm{m} / \mathrm{z})$ : 424.90 .

(5e):IR $\left(\mathrm{KBr}, \mathrm{Cm}^{-1}\right): 3115.43,3078.27(>\mathrm{C}-\mathrm{H}$ stretch, 5-bromo-2-chlorophenoxy) ,2760.72, 2925.92 (>C-H stretch, $\mathrm{CH}_{3}$ groups of tert butyl moiety and $-\mathrm{CH}_{3}$ groups attached to1,2,4,-triazin-4-one), 1693.17 ( $>\mathrm{C}=0,2$,4,-triazin-4-one ), $1510.92(>\mathrm{C}=\mathrm{N}$ stretch),1543.20,1383.33,1360.73( $>\mathrm{C}=\mathrm{C}<$ stretch), 1449.38( $>\mathrm{N}-\mathrm{N}<$ stretch), 1287.56 ( $>\mathrm{C}-\mathrm{S}-$ stretch), 829.40(-C-Cl stretch)and 783.42 (-C-Br stretch). ${ }^{1} \mathrm{H}-\mathrm{NMR}:\left(\mathrm{CDCl}_{3} \delta \mathrm{ppm}\right): 1.532\left(9 \mathrm{H}, \mathrm{s}, 3 \mathrm{CH}_{3}\right.$ groups, tert butyl moiety), $7.458(1 \mathrm{H}, \mathrm{d}, \mathrm{J}=8.8$, of 5-bromo-2-chlorophenoxy moiety ), $7.686(1 \mathrm{H}, \mathrm{d}, \mathrm{J}=2.4$ meta coupling, of 5-bromo-2-chlorophenoxy moiety), $8.446(1 \mathrm{H}, \mathrm{d}, \mathrm{J}=2.4$ meta coupling, of 5-bromo-2-chlorophenoxy moiety). ${ }^{13} \mathrm{C}-\mathrm{NMR}: 27.702\left(3 \mathrm{CH}_{3}\right.$ groups,ter butyl moiety), 38.274 (quaternary $\mathrm{C}$ atom, tert butyl moiety), 121.689, $128.188,132.373,133.657,136.628,138.346$ (6C atoms of 5-bromo-2-chloro phenoxy group),146.789, 156.199, 159.445 (3C atoms thiadiazolotriazin-4-one moiety),161.871 $(>\mathrm{C}=0$ thiadiazolotriazin-4-one). LC-Mass: $\left[\mathrm{M}^{+}+1\right],(\mathrm{m} / \mathrm{z}): 400.92$.

(5f):IR $\left(\mathrm{KBr}, \mathrm{Cm}^{-1}\right): 3115.43,3138.27\left(>\mathrm{C}-\mathrm{H}\right.$ stretch, 2-bromo-5-chloro phenoxy) ,2980.72 (>C-H stretch, $-\mathrm{CH}_{3}$ groups of tert butyl moiety and $-\mathrm{CH}_{3}$ groups attached to1,2,4,-triazin-4-one), $1713.17(>\mathrm{C}=0,2,4$,-triazin-4-one ),1510.92 ( $>\mathrm{C}=\mathrm{N}$ stretch),1543.20,1383.33,1360.73( $>\mathrm{C}=\mathrm{C}<$ stretch), 1449.38( $>\mathrm{N}-\mathrm{N}<$ stretch) , 1287.56 ( $>\mathrm{C}-\mathrm{S}-$ stretch), 829.90(-C-Cl stretch) and 777.42 (-C-Br stretch). ${ }^{1} \mathrm{H}-\mathrm{NMR}:\left(\mathrm{CDCl}_{3} \delta \mathrm{ppm}\right): 1.926\left(9 \mathrm{H}, \mathrm{s}, 3 \mathrm{CH}_{3}\right.$ groups, tert butyl moiety), $7.548(1 \mathrm{H}, \mathrm{d}, \mathrm{J}=8.8$, of 2-bromo-5-chloro phenoxy moiety ), 7.789 ( $1 \mathrm{H}, \mathrm{d}, \mathrm{J}=2.4$ meta coupling, of 2-bromo-5-chloro phenoxy moiety), $8.749(1 \mathrm{H}, \mathrm{d}, \mathrm{J}=2.4$ meta coupling, of 2-bromo-5-chlorophenoxy moiety). ${ }^{13} \mathrm{C}-\mathrm{NMR}: 27.643\left(3 \mathrm{CH}_{3}\right.$ groups,ter butyl moiety), 38.274 (quaternary $\mathrm{C}$ atom, tert butyl moiety), 121.689 , 128.188, 132.373, 133.657, 136.628, 138.346 (6C atoms of 2-bromo-5-chloro phenoxy group),146.789, 156.199, 159.445 (3C atoms thiadiazolotriazin-4-one moiety),160.992(>C $=0$ thiadiazolotriazin-4-one). LC-Mass: [ $\left.{ }^{+}+1\right]$, (m/z): 399.69 .

(5g): IR $\left(\mathrm{KBr}, \mathrm{Cm}^{-1}\right): 3071.33\left(>\mathrm{C}-\mathrm{H}\right.$ stretch, 3,5-dibromo phenoxy), 2955.63 ( $>\mathrm{C}-\mathrm{H}$ stretch, $-\mathrm{CH}_{3}$ groups of tert butyl moiety and $-\mathrm{CH}_{3}$ groups attached to1,2,4,-triazin-4-one), 1710.43( $>\mathrm{C}=0$, 2,4,-triazin-4-one ), 1559.07( $\left.>\mathrm{C}=\mathrm{N}\right)$ ,1524.80, $1360.70 \quad(>\mathrm{C}=\mathrm{C}<$ stretch), $1427.77(>\mathrm{N}-\mathrm{N}<$ stretch), 1264.67 (>C-S- stretch) and 753.29(-C-Br stretch). ${ }^{1} \mathrm{H}-\mathrm{NMR}:\left(\mathrm{CDCl}_{3} \delta \mathrm{ppm}\right): 1.522\left(9 \mathrm{H}, \mathrm{s}, 3 \mathrm{CH}_{3}\right.$ groups, tert butyl moiety), $7.926(1 \mathrm{H}, \mathrm{s}, 3,5$-dibromo phenoxy), $8.046\left(2 \mathrm{H}, \mathrm{s}, 3,5\right.$-dibromo phenoxy ). ${ }^{13} \mathrm{C}-\mathrm{NMR}: 27.659\left(3 \mathrm{CH}_{3}\right.$ groups,ter butyl moiety), 38.346 (quaternary C atom, tert butyl moiety), 124.226, 129.330, 130.113,130.814, 136.932, 138.722 (6C atoms of 3,5dibromo phenoxy group),138.722, 146.787,157.456 (3C atoms thiadiazolotriazin-4-one moiety), $161.28(>\mathrm{C}=0$ thiadiazolotriazin-4-one). LC-Mass: [M+1], (m/z): 444.95 .

(5h):IR ( $\left.\mathrm{KBr}, \mathrm{Cm}^{-1}\right)$ : 3072.46(>C-H stretch, 4- trifluoromethyl phenoxy), 2950.43, 2954.03 (>C-H stretch, $-\mathrm{CH}_{3}$ groups of tert butyl moiety and $-\mathrm{CH}_{3}$ groups attached to1,2,4,-triazin-4-one), 1713.38 ( $>\mathrm{C}=0,2,4$,-triazin-4-one ), 1541.97, $(>\mathrm{C}=\mathrm{N}), 1523.92,1320.50(>\mathrm{C}=\mathrm{C}<$ stretch $), 1458.76(>\mathrm{N}-\mathrm{N}<$ stretch $)$ and $1320.50(>\mathrm{C}-\mathrm{S}-$ stretch $) .{ }^{1} \mathrm{H}-$ NMR: $\left(\mathrm{CDCl}_{3} \delta \mathrm{ppm}\right): 1.9175\left(9 \mathrm{H}, \mathrm{s}, 3 \mathrm{CH}_{3}\right.$ groups, tert butyl moiety), 7.214 $(2 \mathrm{H}, \mathrm{d}, \mathrm{J}=8$, phenoxy ring $), 8.312(2 \mathrm{H}, \mathrm{d}$ , J=8.2 phenoxy ring), 1.423 (1H, s, $\mathrm{CH}$ of propan-2-yloxy). 1.667 (6H,s, $\mathrm{CH} 3$ of propan-2-yloxy). ${ }^{13} \mathrm{C}-\mathrm{NMR}: 27.434$ , $28.234\left(3 \mathrm{CH}_{3}\right.$ groups,ter butyl moiety and $2 \mathrm{C}$ atoms of methyl groups), 38.834 (quaternary $\mathrm{C}$ atom, tert butyl moiety),124.226, 129.330, 130.814 (6C atoms of 3,5-dibromo phenoxy group),138.722, 146.787,157.456 (3C atoms thiadiazolotriazin-4-one moiety),161.28(>C=0 thiadiazolotriazin-4-one). LC-Mass: [ $\left.\mathrm{M}^{+}+1\right],(\mathrm{m} / \mathrm{z}): 344.43$.

(5i):IR $\left(\mathrm{KBr}, \mathrm{Cm}^{-1}\right)$ : 3073.47(>C-H stretch, 4- trifluoromethyl phenoxy), 2960.44, 2924.02 (>C-H stretch, $-\mathrm{CH}_{3}$ groups of tert butyl moiety and $-\mathrm{CH}_{3}$ groups attached to1,2,4,-triazin-4-one), 1693.36 ( $>\mathrm{C}=0$, ,2,4,-triazin-4-one ), 1540.97, ( $>\mathrm{C}=\mathrm{N}), 1513.92,1320.50$ ( $>\mathrm{C}=\mathrm{C}<$ stretch $), 1456.76(>\mathrm{N}-\mathrm{N}<$ stretch $), 1320.50(>\mathrm{C}-\mathrm{S}-$ stretch $), 936.66(-$ C-F stretch) and 1540.97,1513.92 (symmetric and asymmetric stretch of $-\mathrm{NO}_{2}$ group). ${ }^{1} \mathrm{H}-\mathrm{NMR}:\left(\mathrm{CDCl}_{3} \delta\right.$ ppm):1.493(9H, s, $3 \mathrm{CH}_{3}$ groups, tert butyl moiety), $4.410(1 \mathrm{H}, \mathrm{s}, 2$-fluoro-5-nitrophenoxy moiety), $7.492(1 \mathrm{H}, \mathrm{d}$, $\mathrm{J}=8$, of 2-fluoro-5-nitrophenoxy moiety), 7.680(1H, d , J=82-fluoro-5-nitrophenoxy moiety).

${ }^{13}$ C-NMR: $27.629\left(3 \mathrm{CH}_{3}\right.$ groups,ter butyl moiety), 38.209 (quaternary $\mathrm{C}$ atom, tert butyl moiety),122.374, 125.081,126.436, 126.509,129.461, 131.124, (6C atoms of 2-fluoro-5-nitro phenoxy group),137.742, 146.924, 
159.394 (3C atoms thiadiazolotriazin-4-one moiety),162.091( $>\mathrm{C}=0$ thiadiazolotriazin-4-one). LC-Mass: $\left[\mathrm{M}^{+}+1\right],(\mathrm{m} / \mathrm{z}): 349.08$.

(5j): IR (KBr, Cm $\left.{ }^{-1}\right)$ : 3364.20, 3073.43(>C-H stretch, 4- trifluoromethyl phenoxy), 2960.53, 2925.37 (>C-H stretch, $-\mathrm{CH}_{3}$ groups of tert butyl moiety and $-\mathrm{CH}_{3}$ groups attached to1,2,4,-triazin-4-one), 1693.46 ( $>\mathrm{C}=0$, ,2,4,-triazin-4one ),1540.94, $(>\mathrm{C}=\mathrm{N}), 1513.98,1320.73(>\mathrm{C}=\mathrm{C}<$ stretch $), 1456.82(>\mathrm{N}-\mathrm{N}<$ stretch $), 1320.73(>\mathrm{C}-\mathrm{S}-\mathrm{stretch})$ and 936.70(-C-F stretch). ${ }^{1} \mathrm{H}-\mathrm{NMR}:\left(\mathrm{CDCl}_{3} \delta \mathrm{ppm}\right): 1.493\left(9 \mathrm{H}, \mathrm{s}, 3 \mathrm{CH}_{3}\right.$ groups, tert butyl moiety), 4.407(2H,s, $\mathrm{CH}_{2}$ adjacent to 4-(trifluoromethyl) phenoxy moiety), $7.490(2 \mathrm{H}, \mathrm{d}, \mathrm{J}=8,4$-(trifluoromethyl) phenoxy moiety), 7.680 (2H, d, J=8, 4-(trifluoromethyl) phenoxy moiety). ${ }^{13} \mathrm{C}-\mathrm{NMR}$ : $27.631,28.356\left(3 \mathrm{CH}_{3}\right.$ groups, tert butyl moiety and $2 \mathrm{C}$ atoms of $\mathrm{CH}_{2}$ group), 38.251 (quaternary $\mathrm{C}$ atom, tert butyl moiety), 37.299(1C atom of trifluoromethyl group) 122.374, 125.080, 126.443, 126.516, 129.460, 130.482, (6C atoms of trifluoromethyl phenoxy group), 137.725, 146.924, 159.440 (3C atoms thiadiazolotriazin-4-one moiety) $162.536(>\mathrm{C}=0$ thiadiazolotriazin-4-one). LC-Mass: $\left[\mathrm{M}^{+}+1\right],(\mathrm{m} / \mathrm{z}): 368.90$.

(5k):IR ( $\left.\mathrm{KBr}, \mathrm{Cm}^{-1}\right): 3078.27\left(>\mathrm{C}-\mathrm{H}\right.$ stretch, 4-(propan-2-yl)phenoxy) ,2781.14 (>C-H stretch, - $\mathrm{CH}_{3}$ groups of tert butyl moiety and $-\mathrm{CH}_{3}$ groups attached to1,2,4,-triazin-4-one), 1723.17( $>\mathrm{C}=0$, , 2,4,-triazin-4-one ), $1510.92(>\mathrm{C}=\mathrm{N}$ stretch), 1543.20,1386.45,1350.82( >C=C< stretch), 1458.38(>N-N< stretch) , 1297.56 (>C-S- stretch). ${ }^{1} \mathrm{H}-\mathrm{NMR}$ : $\left(\mathrm{CDCl}_{3} \delta \mathrm{ppm}\right): 1.175\left(9 \mathrm{H}, \mathrm{s}, 3 \mathrm{CH}_{3}\right.$ groups, tert butyl moiety), $7.342(1 \mathrm{H}, \mathrm{s},-\mathrm{CH}$ of propan-2-yl)), $8.176(2 \mathrm{H}, \mathrm{d}, \mathrm{J}=8$, 4-(propan-2-yl)phenyl), $8.236\left(2 \mathrm{H}, \mathrm{d}, \mathrm{J}=8,4\right.$-(propan-2-yl)phenyl).0.812(6H,s,-CH $\mathrm{H}_{3}$ groups of propan-2-yl). ${ }^{13} \mathrm{C}$ NMR: 27.894, 27.998. (3 $\mathrm{CH}_{3}$ groups, ter butyl moiety and $2 \mathrm{CH}_{3}$ groups of propan-2-yl ), 38.737 (quaternary $\mathrm{C}$ atom, tert butyl moiety),109.220, 115.818, 123.982, 141.630, 148.078, 152.679 (6C atoms of 4-(propan-2-yl) phenoxy group),157.106, 158.183, 159.912 (3C atoms thiadiazolotriazin-4-one moiety),161.192(>C=0

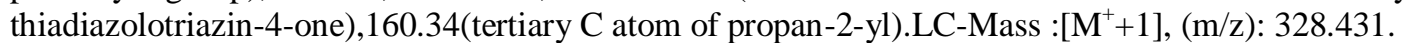

Table 1:- Characterization data of [3-tert-butyl-7-(aryl)-4H-[1,3,4] thiadiazolo[2,3-c][1,2,4]triazin-4-one] (5a-k).

\begin{tabular}{|c|c|c|c|c|c|c|}
\hline \multirow[t]{2}{*}{ SAMPLE } & \multirow[t]{2}{*}{$\mathrm{R}$} & \multirow[t]{2}{*}{$\begin{array}{l}\text { MF } \\
\text { (MW) }\end{array}$} & \multirow[t]{2}{*}{$\begin{array}{l}\text { MP } \\
\left({ }^{0} \mathrm{C}\right)\end{array}$} & \multicolumn{3}{|c|}{$\begin{array}{lrl}\% & \text { COMPOSITION } & \text { FOUND } \\
\text { (CALCULATED) } & \end{array}$} \\
\hline & & & & $\mathrm{C}$ & $\mathrm{H}$ & $\mathrm{N}$ \\
\hline $5 a$ & $-\left(2,3,4-\left(\mathrm{OCH}_{3}\right)_{3}-\mathrm{Ph}\right)$ & $\begin{array}{l}\mathrm{C}_{17} \mathrm{H}_{20} \mathrm{~N}_{4} \mathrm{O}_{4} \mathrm{~S} \\
(376.43)\end{array}$ & $170-173$ & $\begin{array}{l}54.24 \\
(53.23)\end{array}$ & $\begin{array}{l}5.36 \\
(5.29)\end{array}$ & $\begin{array}{l}14.88 \\
(14.66)\end{array}$ \\
\hline $5 b$ & $-\left(2-\mathrm{CH}_{3}, 4-\mathrm{NO}_{2}-\mathrm{Ph}\right)$ & $\begin{array}{l}\mathrm{C}_{15} \mathrm{H}_{15} \mathrm{~N}_{5} \mathrm{O}_{3} \mathrm{~S} \\
(345.37)\end{array}$ & $97-104$ & $\begin{array}{l}52.16 \\
(52.00)\end{array}$ & $\begin{array}{l}4.38 \\
(4.29) \\
\end{array}$ & $\begin{array}{l}20.28 \\
(19.89) \\
\end{array}$ \\
\hline $5 c$ & $-\left(2-\mathrm{C}_{6} \mathrm{H}_{5}-\mathrm{C}_{9} \mathrm{H}_{5} \mathrm{~N}\right)$ & $\begin{array}{l}\mathrm{C}_{23} \mathrm{H}_{21} \mathrm{~N}_{5} \mathrm{OS} \\
(413.49)\end{array}$ & $134-139$ & $\begin{array}{l}66.48 \\
(66.20)\end{array}$ & $\begin{array}{l}5.09 \\
(5.00) \\
\end{array}$ & $\begin{array}{l}16.85 \\
(16.65)\end{array}$ \\
\hline $5 \mathrm{~d}$ & -(Ph-CO-(4-Cl-Ph) & $\begin{array}{l}\mathrm{C}_{21} \mathrm{H}_{17} \mathrm{ClN}_{4} \mathrm{O}_{2} \mathrm{~S} \\
(424.90)\end{array}$ & $149-151$ & $\begin{array}{l}59.36 \\
(60.05)\end{array}$ & $\begin{array}{l}4.03 \\
(4.00)\end{array}$ & $\begin{array}{l}13.19 \\
(13.34)\end{array}$ \\
\hline $5 e$ & $-(2-\mathrm{Cl}, 5-\mathrm{Br}-\mathrm{Ph})$ & $\begin{array}{l}\mathrm{C}_{14} \mathrm{H}_{12} \mathrm{BrClN}_{4} \mathrm{OS} \\
(399.69)\end{array}$ & $103-106$ & $\begin{array}{l}42.07 \\
(42.00)\end{array}$ & $\begin{array}{l}3.03 \\
(3.00)\end{array}$ & $\begin{array}{l}14.02 \\
(14.00)\end{array}$ \\
\hline $5 \mathrm{f}$ & $-(2-\mathrm{Br}, 5-\mathrm{Cl}-\mathrm{Ph})$ & $\begin{array}{l}\mathrm{C}_{14} \mathrm{H}_{12} \mathrm{BrClN}_{4} \mathrm{OS} \\
(399.69)\end{array}$ & $128-132$ & $\begin{array}{l}42.07 \\
(42.00)\end{array}$ & $\begin{array}{l}3.03 \\
(3.00)\end{array}$ & $\begin{array}{l}14.02 \\
(14.00)\end{array}$ \\
\hline $5 \mathrm{~g}$ & $-\left(3,5-(\mathrm{Br})_{2}-\mathrm{Ph}\right)$ & $\begin{array}{l}\mathrm{C}_{14} \mathrm{H}_{12} \mathrm{Br}_{2} \mathrm{~N}_{4} \mathrm{OS} \\
(444.14)\end{array}$ & $156-160$ & $\begin{array}{l}37.86 \\
(38.05)\end{array}$ & $\begin{array}{l}2.72 \\
(3.0)\end{array}$ & $\begin{array}{l}12.61 \\
(12.99)\end{array}$ \\
\hline $5 \mathrm{~h}$ & $-\left(\mathrm{C}_{6} \mathrm{H}_{4}-\mathrm{CO}-\left(\mathrm{CH}_{3}\right)_{2}\right.$ & $\begin{array}{l}\mathrm{C}_{17} \mathrm{H}_{20} \mathrm{~N}_{4} \mathrm{O}_{2} \mathrm{~S} \\
(344.43)\end{array}$ & $119-121$ & $\begin{array}{l}59.28 \\
(59.45)\end{array}$ & $\begin{array}{l}5.85 \\
(5.67)\end{array}$ & $\begin{array}{l}16.27 \\
(16.28)\end{array}$ \\
\hline $5 \mathrm{i}$ & $-\left(2-\mathrm{F}, 5-\mathrm{NO}_{2}-\mathrm{Ph}\right)$ & $\begin{array}{l}\mathrm{C}_{14} \mathrm{H}_{12} \mathrm{FN}_{5} \mathrm{O}_{3} \mathrm{~S} \\
(349.34)\end{array}$ & $110-112$ & $\begin{array}{l}48.13 \\
(48.24)\end{array}$ & $\begin{array}{l}3.46 \\
(3.54) \\
\end{array}$ & $\begin{array}{l}20.05 \\
(19.98) \\
\end{array}$ \\
\hline $5 \mathrm{j}$ & $-\mathrm{CH}_{2}-\left(4-\mathrm{CF}_{3}-\mathrm{Ph}\right)$ & $\begin{array}{l}\mathrm{C}_{16} \mathrm{H}_{15} \mathrm{~F}_{3} \mathrm{~N}_{4} \mathrm{OS} \\
(368.37)\end{array}$ & $160-169$ & $\begin{array}{l}52.17 \\
(52.23)\end{array}$ & $\begin{array}{l}4.10 \\
(3.99)\end{array}$ & $\begin{array}{l}15.21 \\
(15.23)\end{array}$ \\
\hline $5 \mathrm{k}$ & $-\mathrm{C}_{6} \mathrm{H}_{4}-\mathrm{C}-\left(\mathrm{CH}_{3}\right)_{2}$ & $\begin{array}{l}\mathrm{C}_{17} \mathrm{H}_{20} \mathrm{~N}_{4} \mathrm{OS} \\
(328.431)\end{array}$ & $88-91$ & $\begin{array}{l}62.17 \\
(62.18)\end{array}$ & $\begin{array}{l}6.14 \\
(6.16)\end{array}$ & $\begin{array}{l}17.06 \\
(17.07)\end{array}$ \\
\hline
\end{tabular}

\section{Biological Activity:-}

Anti-bacterial activity:-

The novel compounds (5a-k) were screened for in vitro antimicrobial activity by disc diffusion method (zone of inhibition test) using ciprofloxacin a antibiotic as a reference standard against two gram positive (Staphylococcus aureus (MTCC-7443), Bacillus subtilius (MTCC-441)) and two gram negative (Escherichia coli (MTCC-725), 
Klebsiella pneumonia (MTCC-1739)).The micro-organism were collected from the institute of microbial technology, Chandigarh, India. The anti-bacterial activity was carried out according to detailed procedure ${ }^{22}$.

The colonies of the microbial strains were inoculated on nutrient agar plates with the help of sterile loop and visually adjusted the turbidity with broth to broth to match that of $0.5 \mathrm{McFarland}$ standards. The excess of the inoculums was removed by rotating the sterile swab dipped in to the inoculum against the wall of the tube against it approximately $60^{\circ} \mathrm{C}$ between streaking, the procedure is repeated three times to ensure even distribution. After 3 mins sterile discs of the size $6 \mathrm{~mm}$ diameter were aseptically impregnated with the test compounds at a concentration $50 \mu \mathrm{g} / \mathrm{ml}$.The plates were incubated at $37^{\circ} \mathrm{C}$ for $24 \mathrm{~h}$. The compounds that produce distinct circular zones of inhibition around the discs .the diameter of clear zone indicate the anti-bacterial activity.

The anti-bacterial activity screening revealed that the test compounds exhibited good to moderate activity, a few of the tested compounds has limited or no sensitivity towards certain strains of reference bacterial strain used. Among the test samples the compounds $5 \mathrm{~g}$ and $5 \mathrm{~h}$ were found to be extremely sensitive towards both gram positive and gram negative bacteria. Whereas the compound $5 \mathrm{c}$ and $5 \mathrm{~b}$ were sensitive only towards one strains of bacteria i.e. $5 \mathrm{c}$ was sensitive towards only gram negative bacteria and showed maximum inhibition in case of E.coli bacterial strain but the compound $5 \mathrm{~b}$ was sensitive to B. subtilius out of the two bacterial strains used and hence can be recommended as drug candidates for the diseases caused by bacteria. The results are tabulated in Table 2.2.

\section{Anti -Tubercular activity:-}

In the present study the synthesized compounds [3-tert-butyl-7-(aryl)-4H-[1,3,4]thiadiazolo[2,3-c][1,2,4]triazin-4one] (22a-k) were screened for in vitro antituberculosis activity by Micro plate Alamar Blue Assay Method(MABA)(MIC Test) according to standard procedure ${ }^{23}$.The reference drugs such as Streptomycin, Ciprofloxacin and Pyrazinamide were used as standards for the study .

The 96 wells plate of outer perimeter was inoculated with $200 \mu \mathrm{l}$ of sterile water and $100 \mu \mathrm{l}$ of middle brook $7 \mathrm{H} 9$ broth and serial dilution of compound were made directly on plate The final drug concentration tested were 100 to $3.12 \mu \mathrm{g} / \mathrm{ml}$, the plates were sealed with parafilm and incubated at $37^{\circ} \mathrm{C}$ for 5 days. Later $25 \mu \mathrm{l}$ of freshly prepared mixture of Alamar Blue reagent and10\%tween 80 in 1:1 ratio was added and incubated for 24 hours .A blue colour in the well was interpreted as no bacterial growth and pink colour as bacterial growth. The antibiotic drugs such as pyrazinamide, streptomycin and ciprofloxacin were used as reference standard, whose standard values are $3.12 \mu \mathrm{g} / \mathrm{ml}, 6.25 \mu \mathrm{g} / \mathrm{ml}$ and $3.125 \mu \mathrm{g} / \mathrm{ml}$ respectively.

All the target compounds exhibited good Anti-TB activity. Among the tested compounds 5e showed excellent, 5c moderate and remaining compounds exhibited good Anti- TB activity.

The relative potency indicates that novel compounds (5a-k) tested in the present study are not as effective as that of pyrazinamide, streptomycin and ciprofloxacin drugs but 5e may be considered as Anti $-\mathrm{TB}$ agent as its value coincides with the values of standard drugs. The results are discussed in Table 2.3 .

Table 2.2:- Anti-bacterial activity of [3-tert-butyl-7-(aryl)-4H-[1,3,4]thiadiazolo[2,3-c][1,2,4]triazin-4-one] (5a-k) against Gram positive and Gram negative Bacteria by disc diffusion method(ZOI test).

\begin{tabular}{|c|c|c|c|c|}
\hline \multirow[t]{3}{*}{ Samples $75 \mu \mathrm{g} / \mathrm{ml}$} & \multicolumn{4}{|c|}{ Diameter of zone inhibition $(\mathrm{mm} \pm \mathrm{SD})^{A}$} \\
\hline & \multicolumn{2}{|c|}{ Gram positive bacteria } & \multicolumn{2}{|c|}{ Gram negative bacteria } \\
\hline & Staphylococcus aureus & Bacillus subtilius & Escherichia coli & Klebsiella pneumonia \\
\hline $5 \mathrm{a}$ & $11 \mathrm{~mm}$ & $12 \mathrm{~mm}$ & $16 \mathrm{~mm}$ & $12 \mathrm{~mm}$ \\
\hline $5 \mathrm{~b}$ & $10 \mathrm{~mm}$ & $20 \mathrm{~mm}$ & $12 \mathrm{~mm}$ & $19 \mathrm{~mm}$ \\
\hline $5 \mathrm{c}$ & $18 \mathrm{~mm}$ & $15 \mathrm{~mm}$ & $30 \mathrm{~mm}$ & $20 \mathrm{~mm}$ \\
\hline $5 \mathrm{~d}$ & $16 \mathrm{~mm}$ & $11 \mathrm{~mm}$ & $10 \mathrm{~mm}$ & $12 \mathrm{~mm}$ \\
\hline $5 \mathrm{e}$ & $16 \mathrm{~mm}$ & $13 \mathrm{~mm}$ & $17 \mathrm{~mm}$ & $18 \mathrm{~mm}$ \\
\hline $5 f$ & $11 \mathrm{~mm}$ & $9 \mathrm{~mm}$ & $\mathrm{R}$ & $19 \mathrm{~mm}$ \\
\hline $5 \mathrm{~g}$ & $22 \mathrm{~mm}$ & $28 \mathrm{~mm}$ & $29 \mathrm{~mm}$ & $17 \mathrm{~mm}$ \\
\hline $5 \mathrm{~h}$ & $21 \mathrm{~mm}$ & $22 \mathrm{~mm}$ & $26 \mathrm{~mm}$ & $24 \mathrm{~mm}$ \\
\hline $5 \mathrm{i}$ & $10 \mathrm{~mm}$ & $14 \mathrm{~mm}$ & $12 \mathrm{~mm}$ & $16 \mathrm{~mm}$ \\
\hline $5 j$ & $12 \mathrm{~mm}$ & $16 \mathrm{~mm}$ & $16 \mathrm{~mm}$ & $16 \mathrm{~mm}$ \\
\hline $5 \mathrm{k}$ & $13 \mathrm{~mm}$ & $13 \mathrm{~mm}$ & $17 \mathrm{~mm}$ & $17 \mathrm{~mm}$ \\
\hline Ciprofloxacin & $26 \mathrm{~mm}$ & $30 \mathrm{~mm}$ & $32 \mathrm{~mm}$ & $28 \mathrm{~mm}$ \\
\hline
\end{tabular}


Note:

${ }^{\mathrm{A}}$ Mean values of 3 trails.

' 0 ' indicates no sensitivity (zone of inhibition $<7 \mathrm{~mm}$ ).

Ref.Std: Ciprofloxacin $(10 \mu \mathrm{g} / \mathrm{disc})$.

Table 2.3:- Anti-tubercular activity of [3-tert-butyl-7-(aryl)-4H-[1,3,4]thiadiazolo[2,3-c][1,2,4]triazin-4-one] (5a-k) by Micro plate Alamar Blue Assay Method (MABA) (MIC Test).

\begin{tabular}{|l|l|l|l|l|l|l|}
\hline & 100 & 50 & 25 & 12.5 & 6.25 & 3.12 \\
SAMPLES & $\mu \mathrm{g} / \mathrm{ml}$ & $\mu \mathrm{g} / \mathrm{ml}$ & $\mu \mathrm{g} / \mathrm{ml}$ & $\mu \mathrm{g} / \mathrm{ml}$ & $\mu \mathrm{g} / \mathrm{ml}$ & $\mu \mathrm{g} / \mathrm{ml}$ \\
\hline $5 \mathrm{a}$ & $\mathrm{S}$ & $\mathrm{S}$ & $\mathrm{R}$ & $\mathrm{R}$ & $\mathrm{R}$ & $\mathrm{R}$ \\
\hline $5 \mathrm{~b}$ & $\mathrm{~S}$ & $\mathrm{~S}$ & $\mathrm{R}$ & $\mathrm{R}$ & $\mathrm{R}$ & $\mathrm{R}$ \\
\hline $5 \mathrm{c}$ & $\mathrm{S}$ & $\mathrm{S}$ & $\mathrm{S}$ & $\mathrm{R}$ & $\mathrm{R}$ & $\mathrm{R}$ \\
\hline $5 \mathrm{~d}$ & $\mathrm{~S}$ & $\mathrm{~S}$ & $\mathrm{R}$ & $\mathrm{R}$ & $\mathrm{R}$ & $\mathrm{R}$ \\
\hline $5 \mathrm{e}$ & $\mathrm{S}$ & $\mathrm{S}$ & $\mathrm{S}$ & $\mathrm{S}$ & $\mathrm{S}$ & $\mathrm{S}$ \\
\hline $5 \mathrm{f}$ & $\mathrm{S}$ & $\mathrm{S}$ & $\mathrm{R}$ & $\mathrm{R}$ & $\mathrm{R}$ & $\mathrm{R}$ \\
\hline $5 \mathrm{~g}$ & $\mathrm{~S}$ & $\mathrm{~S}$ & $\mathrm{R}$ & $\mathrm{R}$ & $\mathrm{R}$ & $\mathrm{R}$ \\
\hline $5 \mathrm{~h}$ & $\mathrm{~S}$ & $\mathrm{~S}$ & $\mathrm{R}$ & $\mathrm{R}$ & $\mathrm{R}$ & $\mathrm{R}$ \\
\hline $5 \mathrm{i}$ & $\mathrm{S}$ & $\mathrm{S}$ & $\mathrm{R}$ & $\mathrm{R}$ & $\mathrm{R}$ & $\mathrm{R}$ \\
\hline $5 \mathrm{j}$ & $\mathrm{S}$ & $\mathrm{S}$ & $\mathrm{R}$ & $\mathrm{R}$ & $\mathrm{R}$ & $\mathrm{R}$ \\
\hline $5 \mathrm{k}$ & $\mathrm{S}$ & $\mathrm{S}$ & $\mathrm{R}$ & $\mathrm{R}$ & $\mathrm{R}$ & $\mathrm{R}$ \\
\hline
\end{tabular}

NOTE: S-Sensitive ; R-Resistant

Strain: M.tuberculosis (H37 RV strain)

Reference standard: Streptomycin- $6.25 \mu \mathrm{g} / \mathrm{ml}$,

Ciprofloxacin-3.12 $\mu \mathrm{g} / \mathrm{ml}$,

Pyrazinamide- $3.12 \mu \mathrm{g} / \mathrm{ml}$

\section{Conclusion:-}

The investigation of the antibacterial and anti-tubercular activity of the synthesized target compounds revealed that these compounds show moderate activity towards both the screening test. The compounds $5 \mathrm{~g}$ and $5 \mathrm{~h}$ showed relativity high activity against the standards the compound 5e and 5c showed excellent activity and moderate activity against the three reference standards used the prominent biological activity was shown the compounds with the electronegative elements like Chloro, Bromo and Nitro groups as substituents. A further study of these compounds with special reference to therapeutic index for the drug is going on.

\section{Acknowledgement:-}

The authors express their heartfelt thanks to, The head ,NMR research center ,SAIF - cochin-22 for 1H-NMR,13CNMR spectroscopy and FT-IR analysis, Syngenta Goa for Mass spectroscopy, Maratha mandal's NGH institute of dental sciences and research center, Belgaum for Microbiological activities

\section{References:-}

1. Sraa A M., Synthesis, antimicrobial evaluation and spectroscopic characterization of novel imidazolone, triazole and triazinone derivatives, Spectrochimica Acta Part A: Molecular and Biomolecular Spectroscopy .96:898905.(2012).

2. Zhenhua L., Baoquan C., Shuangyan H., Yanping S., Yuming L., Caiwen Li., Synthesis and antitumorevaluation of 1,3,4-thiadiazole-containing benzisoselenazolone derivatives, Bioorg. Med. Chem. Lett.22:31913193. (2012).

3. Shankar G A., Kallanagouda R A., Pranali V S., Sagar M C., Dilip H D., Amol S S., Novel imidazo[2,1b][1,3,4]thiadiazole carrying rhodanine-3-acetic acid as potential antitubercular agents, Bioorg. Med. Chem. Lett.22:1917-1921. (2012).

4. Tarik El-S A., Synthesis and antibacterial activity of some new thiadiaza / triazaphospholes , thiadiaza/triaza/tetrazaphosphinines and thiadiaza / tetrazaphosphepines containing 1,2,4-triazinone moiety, Eur: J. Med. Chem.44:4539-4546. (2009).

5. Darpan K., Suroor A K., Gita C., Design \& synthesis of 2-(substituted aryloxy)-5-(substituted benzylidene)-3phenyl-2,5-dihydro-1H-[1,2,4] triazin-6-one as potential anticonvulsant agents, Eur: J. Med. Chem. 45:39603969. (2010). 
6. Małgorzata S., Jolanta R., Krzyszt S., Synthesis, structure elucidation and in vitro anticancer activities of novel derivatives of diethyl (2E)-2-[(2E)-(1-arylimidazolidin-2-ylidene)hydrazono]succinate and ethyl (4-oxo-8-aryl4,6,7,8-tetrahydroimidazo[2,1-c] [1,2,4] triazin-3-yl ) acetate ,Bioorg. Med. Chem.21:7465-7480. (2013).

7. Fabian K., Hans M D., Hans H R., Petra F., Synthesis and characterization of novel 1,2,4-triazine derivatives with antiproliferative activity, Bioorg. Med. Chem.18:1816-1821. (2010).

8. Krzyszt S., Kazimierz P., Małgorzata S., Martyna K S., Anna E K., Izabela D., Crystal structure, antitumour and antimetastatic activities of disubstituted fused 1,2,4-triazinones, Bioorg. Med. Chem. Lett.19:5095-5100. (2009).

9. Zachary K S., Sahaja A., Andrew B., James P D., Todd R E., Jennifer F., Anthony M G., Gabrielle H., Yu Li., Ann C K., Michael M., David Y S., Mark S., Judy M S., Steven S., Jeffrey W., Julie Q H., Amy S Z., Klaus K., Discovery of triazolinone non-nucleoside inhibitors of HIV reverse transcriptase, Bioorg. Med. Chem. Lett.18:4348-4351. (2008)

10. Mohammad Y., Riaz A K., Bahar A., Syntheses and anti-depressant activity of 5-amino-1, 3, 4-thiadiazole-2thiol imines and thiobenzyl derivatives, Bioorg. Med. Chem. Lett.16:8029-8034. (2008).

11. Mohd A., Kumar S., Synthesis and anti-inflammatory, analgesic, ulcerogenic and lipid peroxidation activities of some new 2-[(2,6-dichloroanilino) phenyl]acetic acid derivatives, Eur: J. Med. Chem. 39:535-545.(2004).

12. Tarik E A., Synthesis and Fungicidal Activity of Some New 4H-Chromen-4-ones Containing Some 1,3Thiazole, 1,3-Thiazine, 1,2,4-Triazole and 1,2,4-Triazine Moieties, Phosphorous ,Sulfur ,Silicon Relat. Elem.182:1717-1726. (2007).

13. Holla B S., Gonsalves R., Sarojini B K., Shenoy S., Synthesis of some new biologically active thriazinothiadiazinones, Ind. J. Chem. 40B:475.(2001).

14. Prakash A C., Prashantha N., Jagadeesh P D., Sujayraj R S., Sharath K C., Kumara C., Ramya N., Sandya Kumari M V., Guruprasad K., Satish Kumar A., Synthesis of novel thiadiazolotriazin-4-ones and study of their mosquito-larvicidal and antibacterial properties, Eur: J. Med. Chem.84:194-199.(2014).

15. Gerhard B., Ulrich B H., Hans. K., (Bayer A-G.)., Synthesis of novel thiadiazolotriazin-4-ones and study of their mosquito-larvicidal and antibacterial properties, Ger. Offen. DE 3, 009, 043 (1980),Chem. Abstr.96 :6764.(1982).

16. Krauz E., Findeisen K., Fiege H., Ene L., Schmidt R R., (Bayer A-G.)., Synthesis of novel thiadiazolotriazin-4ones and study of their mosquito-larvicidal and antibacterial properties, Ger. Offen. DE3, 323, 934 (1983), Chem. Abstr.102:18506. (1985).

17. Beat B., Hans T (Ciba-Geigy A-G)., Eur. Pat. Appl., Ep 150, 677., Synthesis of novel thiadiazolotriazin-4-ones and study of their mosquito-larvicidal and antibacterial properties, Chem. Abstr.104:19607.(1986).

18. Jagadeesh P D., Karthikeyan M S., Prakash B K., Boja P,. Shivarama B H., Suchetha. N.K., Synthesis of some thiadiazolotriazinone derivatives as possible antimicrobial agents, Phosphorous, Sulfur, Silicon Relat. Elem. 182:1083-1091. (2007).

19. Dornow D., Mazel H., Marxd P., Ber.97:2173. (1964).

20. M.K.Shivananda., "Studies on synthesis, characterization and biological activities of some aryl furan heterocycles", PhD Thesis, Mangalore university India.(1999).

21. Prakash A C., Prashantha N., Jagadeesh P D., Sujayraj RS., Sharath K C., Kumara C., Ramya N,. Sandya Kumari M V., Guruprasad K., Satish Kumar A., Synthesis of novel thiadiazolotriazin-4-ones and study of their mosquito-larvicidal and antibacterial Properties, Eur: J. Med. Chem. 84:194-199. (2014).

22. Hasan C M., Begum S N., Illias M., Husain A., J.bot.17:135-137. (1988).

23. Bauer AW., Kirby W M M., Sherris J C., Turck M., Am.j.Clin.pathology .493. (1966). 